\title{
Connaissance du VIH/sida, perception du risque et comportements de prévention
}

\author{
Nathalie BELTZER ${ }^{1}$ et Isabelle GRÉMY ${ }^{2}$
}

\begin{abstract}
Résumé
Face au sida, les résultats des enquêtes KABP permettent de comprendre si l'évolution des comportements de prévention chez les femmes au cours des 15 dernières années est la résultante d'une modification des perceptions du risque individuel ou du risque social. Ils montrent aussi que l'adaptation au risque de contamination par le VIH diffère entre les femmes et les hommes. Les femmes, notamment les jeunes et les moins diplômées, rapportent plus fréquemment ne pas avoir utilisé de préservatif. Les résultats de ces enquêtes soulignent la difficulté de certaines femmes, aujourd'hui, à imposer l'utilisation du préservatif, alors qu'elles ont une bonne connaissance de la maladie et une perception du risque individuel de contamination plus élevée que les hommes. Elles se retrouvent plus fréquemment dans l'obligation d'accepter des rapports sexuels sans préservatif lorsque leur partenaire ne souhaite pas en utiliser. Elles adoptent en revanche des stratégies alternatives plus fréquentes (telles que le choix du partenaire, la réduction de leur nombre) qui, contrairement aux hommes, ne semblent pas se substituer à l'utilisation du préservatif.
\end{abstract}

Mots clés : VIH, perception du risque, comportement à risque, prévention.

Les premières années, la catastrophe sanitaire et sociale qu'a représenté l'épidémie du VIH/sida en France a entrainé une mobilisation exceptionnelle des acteurs de la société. Les pouvoirs publics y ont pris leur part, notamment par une prise en charge spécifique des personnes séropositives, une ré-organisation de l'offre de soins et la mise en place de campagnes de prévention.

Comme le montrent les enquêtes en population générale (voir plus loin) ou celles menées auprès de populations ciblées, une adaptation très rapide et généralisée des comportements sexuels a été enregistrée, notamment à travers une forte augmentation de l'utilisation des préservatifs.

L'arrivée des traitements antirétroviraux, en 1996, a considérablement amélioré le pronostic de la maladie, mais s'est accompagnée d'un relâchement des comportements de pré-

Observatoire régional de santé d'île-de-France Id. vention. Ainsi, le rapport publié par l'Institut national de veille sanitaire (InVS) en mars 2007 indique qu'il y a encore 6000 à 7000 découvertes de séropositivités par an [1]. On assiste à une recrudescence des maladies sexuellement transmissibles, voire à une résurgence de certaines d'entres elles comme la syphilis [2], ou à l'apparition de certaines infections jusque-là inconnues dans nos contrées, comme la lymphogranulomatose vénérienne [3]. La reprise de l'épidémie chez les homosexuels masculins, crainte au cours de ces dernières années, est aujourd'hui confirmée avec, parallèlement, une évolution progressive du profil des personnes atteintes par le $\mathrm{VIH} /$ sida. La proportion de personnes hétérosexuelles infectées par le VIH, essentiellement d'origine d'Afrique sub-saharienne, augmente et on assiste à une féminisation de la maladie. Le rapport de l'InVS [1] constate que les femmes représentaient $21 \%$ des cas de sida en 1996, alors que cette proportion était de $33 \%$ en 2005. Les femmes représentent $40 \%$ des découvertes de nouvelles séropositivités. Leur plus jeune âge est également un facteur préoccupant.

Les femmes apparaissent donc particulièrement vulnérables face au VIH. Si cette situation peut s'expliquer en partie par une probabilité deux fois plus élevée que pour les hommes d'être contaminées lors d'un rapport sexuel non protégé, les facteurs sociaux semblent aussi en cause (inégalité des conditions socioéconomiques, normes culturelles en leur défaveur) et peuvent expliquer la moindre protection des femmes vis-à-vis du risque de transmission.

La représentation sociale du $\mathrm{VIH} /$ sida et son évolution au fil des enquêtes est très similaire chez les hommes et les femmes. En effet, hommes et femmes partagent le même niveau de connaissances des modes de transmission et de protection, les mêmes attitudes à l'égard des personnes séropositives et des croyances identiques [4]. On observe en revanche des comportements sexuels et préventifs différents. Les femmes ont ainsi davantage recours au test de dépistage, elles sont moins nombreuses à déclarer plusieurs partenaires sexuels au cours des douze derniers mois mais disent plus fréquemment ne pas avoir utilisé de préservatif.

La question se pose donc de savoir ce qui conduit les femmes à adopter des comportements de prévention différents de ceux des 
hommes, alors qu'ils partagent une même représentation sociale de la maladie. Une perception élevée du risque subjectif est un des éléments conduisant à l'adoption de comportements de prévention, ce risque subjectif regroupant tout autant le risque perçu pour soi (risque individuel) ou pour la société (risque social) [5].

L'objectif est ici de savoir si, à la lumière des résultats des enquêtes KABP, l'évolution des comportements de prévention chez les femmes est la résultante d'une modification de la représentation sociale du sida ainsi que des perceptions du risque individuel ou du risque social. II s'agit aussi de montrer en quoi l'adaptation au risque subjectif diffère entre les femmes et les hommes.

Pour ce faire, nous proposons, à partir des enquêtes KABP effectuées en France métropolitaine: de suivre l'évolution des connaissances sur la maladie des femmes interrogées; d'étudier leur attitude vis-à-vis des malades et des personnes infectées ; d'étudier leur perception du risque individuel et social de la contamination par le VIH ; enfin, d'analyser les logiques qui soustendent la mise en œuvre de certaines pratiques préventives. Ces indicateurs seront comparés à ceux des hommes. Nous verrons ensuite comment les femmes s'adaptent au risque de contamination du VIH, en choisissant des stratégies alternatives au préservatif et en tenant compte du cadre relationnel et affectif dans lequel se déroulent le rapport sexuel et la négociation de l'utilisation du préservatif.

\section{Bref rappel méthodologique des enquêtes KABP en métropole}

Les enquêtes KABP (Knowledge, Attitudes, Beliefs and Practices) de 1992, 1994, 1998, 2001 et 2004 ont toutes été réalisées par téléphone à partir d'un échantillon aléatoire de numéros [4]. Pour rendre la structure similaire à celle de la population vivant en France, l'échantillon a d'abord été pondéré par la taille du ménage puis redressé sur les caractéristiques de sexe, d'âge, d'activité professionnelle et de vie en couple.

Au total, les 5376 femmes et 4451 hommes interrogés au cours de ces cinq vagues d'enquête onten moyenne 36 ans (tableau 1). Une proportion identique (environ deux tiers) de femmes et d'hommes déclare vivre en couple, proportion stable au cours des enquêtes. Les femmes sont davantage diplômées que les hommes : $61 \%$ d'entre elles déclarent un diplôme équivalent ou supérieur au baccalauréat versus $54 \%$ des hommes en 2004. Les femmes comme les hommes sont plus nombreux en 2004 qu'en 1992 à être titulaires d'un diplôme supérieur au baccalauréat.

L'analyse des résultats a été effectuée avec le logiciel Stata.

\section{Connaissance de la maladie, perception du risque de contamination et comportements préventifs des femmes}

De nombreux auteurs ont déjà étudié les liens entre les connaissances, les attitudes, les croyances et les comportements face au $\mathrm{VIH} /$ sida, mais peu de ces études portent spécifiquement sur les femmes.

Tableau 1

Âge, diplôme et statut matrimonial des femmes et des hommes inclus dans les enquêtes KABP de 1992, 1994, 1998, 2001 et 2004

\begin{tabular}{|c|c|c|c|c|c|c|c|c|c|c|}
\hline & \multicolumn{2}{|c|}{1992} & \multicolumn{2}{|c|}{1994} & \multicolumn{2}{|c|}{1998} & \multicolumn{2}{|c|}{2001} & \multicolumn{2}{|c|}{2004} \\
\hline & $\mathbf{F}$ & H & $\mathbf{F}$ & $\mathrm{H}$ & $F$ & $\mathrm{H}$ & $\mathrm{F}$ & $\mathrm{H}$ & $F$ & H \\
\hline Effectif & 802 & 673 & 661 & 523 & 886 & 848 & 1418 & 1124 & 1609 & 1283 \\
\hline \multicolumn{11}{|l|}{ Age en année } \\
\hline Moyenne & 35,2 & 34,8 & 35,1 & 35 & 34,8 & 35,4 & 36,3 & 36,4 & 35,6 & 36,9 \\
\hline Probabilité & NS & NS & NS & NS & NS & & & & & \\
\hline \multicolumn{11}{|l|}{ Diplôme } \\
\hline Aucun & 20,3 & 14,3 & 17,3 & 9,9 & 11,9 & 13,7 & 12,8 & 8,9 & 9 & 8 \\
\hline CAP/CEP & 36,3 & 44,1 & 38,4 & 42,7 & 35,5 & 42,9 & 30,6 & 37,9 & 29,9 & 38 \\
\hline BAC & 19 & 16,5 & 19,1 & 23 & 22,8 & 17,9 & 22,8 & 20,2 & 22,9 & 19,8 \\
\hline Supérieur au BAC & 24,4 & 25,1 & 25,2 & 24,4 & 29,9 & 25,5 & 33,8 & 33 & 38,2 & 34,2 \\
\hline Probabilité & $\mathrm{S}^{\star}$ & $\mathrm{S}$ & S & S & $\mathrm{S}$ & & & & & \\
\hline \multicolumn{11}{|c|}{ En couple/cohabitant } \\
\hline Oui & 68,3 & 64,4 & 68,2 & 62,2 & 66,7 & 66 & 66,6 & 63,7 & 66,1 & 63,6 \\
\hline Non & 31,7 & 35,6 & 31,8 & 37,8 & 33,3 & 34 & 33,4 & 36,3 & 33,9 & 36,4 \\
\hline Probabilité & $\mathrm{NS}^{\star *}$ & NS & NS & NS & NS & & & & & \\
\hline
\end{tabular}

Champs : femmes et hommes âgé-es de 18 à 54 ans répondants aux enquêtes de 1992, 1994, 1998, 2001 et 2004.

Test du $\chi 2$ simple sur les effectifs redressés.

" Significatif pour un $p<0,05$; ${ }^{* *}$ Non significatif. 


\section{Une dégradation du niveau de connaissance de la maladie et un essoufflement de la mobilisation chez les femmes jeunes non diplômées}

La représentation sociale du sida sera ici appréhendée à travers le niveau de connaissance de la maladie, l'acceptation des personnes séropositives et l'intérêt pour les campagnes de prévention.

Les femmes connaissent bien les modes de transmission du VIH et les moyens pour s'en protéger, puisque plus de $99 \%$ d'entre elles savent que le virus du sida peut se transmettre par une injection de drogue ou lors de rapports sexuels sans préservatif. Elles sont 93,4 \% à considérer qu'utiliser un préservatif masculin est efficace pour se protéger. Enfin, deux tiers d'entre elles connaissent l'existence des traitements antirétroviraux, proportion en augmentation par rapport à 2001 (elles étaient alors $60,3 \%)$.

Comme pour les hommes, certains mécanismes de transmission restent toutefois moins bien maîtrisés en 2004 (tableau 2). Or, croire par exemple que le sida se transmet par les toilettes publi- ques ou par une piqûre de moustique est associé à une plus faible acceptation des personnes séropositives [6]. Ce sont les femmes les plus jeunes qui connaissent le moins bien la maladie aujourd'hui. Alors que jusqu'en 1998, les femmes âgées de 18 à 24 ans maîtrisaient mieux que leurs aînées les mécanismes de transmission du virus du sida, elles ont depuis un niveau global de la connaissance inférieur, notamment par rapport aux femmes de 25 à 39 ans. Les femmes les plus jeunes sont plus nombreuses à penser que " se laver après l'acte sexuel " est tout à fait ou plutôt efficace pour se protéger du sida ( $16,7 \%$ versus $11 \%$ de celles de 25-39 ans) et moins nombreuses à connaître l'existence des traitements antirétroviraux.

Les mécanismes de transmission et de prévention sont également moins bien maîtrisés par les femmes les moins diplômées, et ce quelle que soit l'année de l'enquête. Un quart des femmes sans diplôme ou titulaires d'un CEP ou CAP disait, en 2004, que le virus du sida peut se transmettre dans les toilettes publiques; elles sont moins d'une sur sept lorsqu'elles possèdent au moins le baccalauréat. Les femmes de 18-24 ans sans diplôme ou titulaires d'un CAP/CEP sont également quatre fois plus nombreuses

Tableau 2

Connaissance des modes de transmission et des moyens de protection et perception du risque de contamination chez les femmes et les hommes en 1992, 1994, 1998, 2001 et 2004

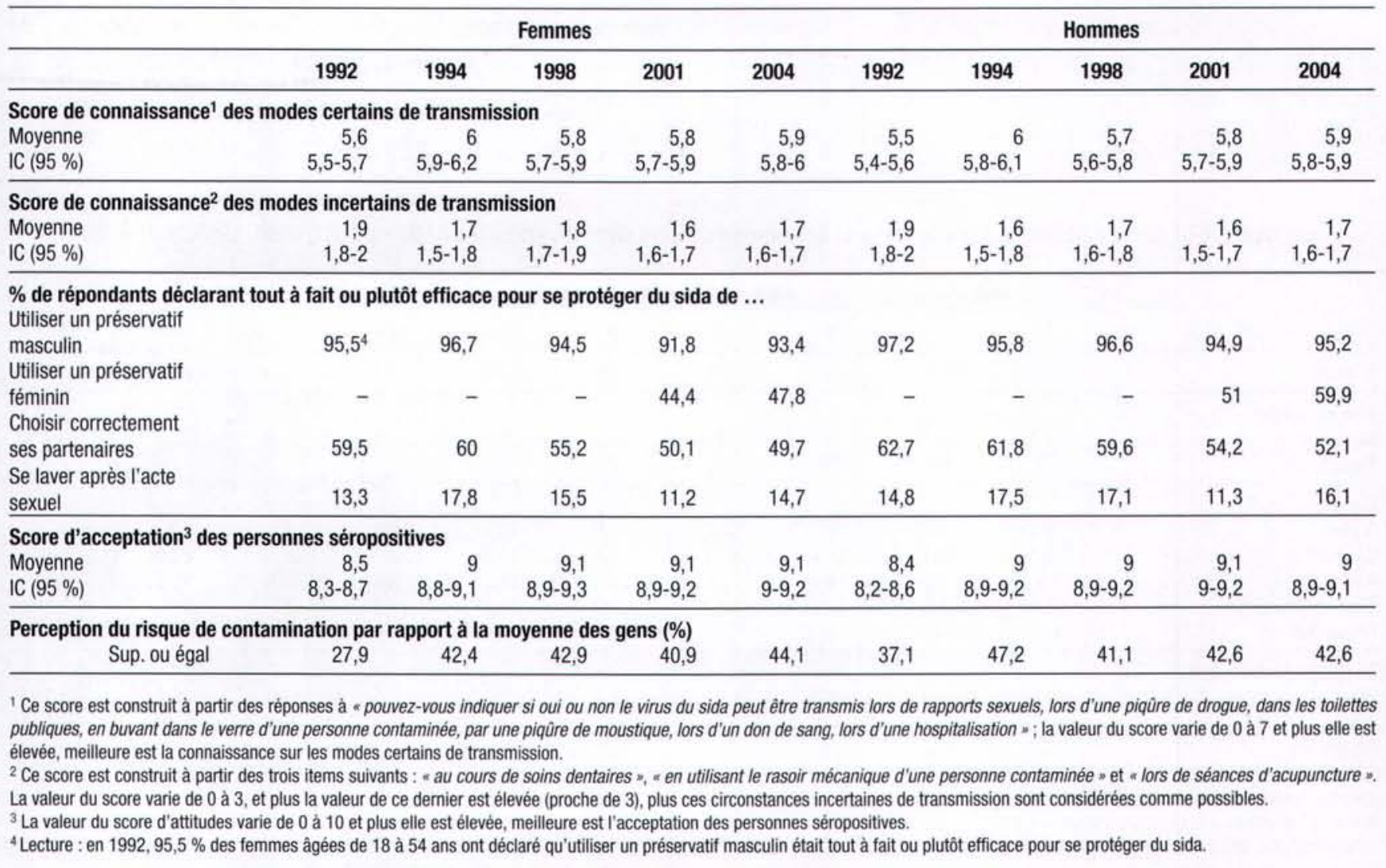


que les plus diplômées à penser que "se laver après l'acte sexuel " est un moyen efficace pour se protéger du sida ( $34,7 \%$ contre $9,1 \%$ des plus diplômées) ; elles étaient respectivement $9,7 \%$ et $4,9 \%(p=0,3)$ en 2001 .

Les femmes connaissent autant que les hommes la maladie, ses mécanismes de transmission et de protection. Comme pour les hommes, cette connaissance s'est détériorée depuis 1998. La détérioration concerne plus particulièrement les jeunes et les moins diplômées. Aussi ce sont aujourd'hui les jeunes et les moins diplômé-es qui maîtrisent le moins bien les mécanismes de transmission.

Les scientifiques et les acteurs de terrain expliquent le maintien, voire le retour, de pratiques à risque dans certains groupes de la population par le moindre intérêt porté au sida. Cet essoufflement de la mobilisation peut être mesuré par l'évolution de certains indicateurs, qui montrent, par exemple, une tolérance plus faible à l'égard des personnes séropositives, un désintérêt croissant pour les campagnes de prévention et une moindre visibilité du sida.

Les femmes (comme les hommes) restent en grande majorité toujours tolérantes à l'égard des personnes séropositives. Depuis la première enquête, environ $90 \%$ des femmes accepteraient de travailler avec une personne séropositive ou de continuer à la fréquenter. Elles sont toutefois, comme les hommes, moins nombreuses à accepter de laisser leurs enfants ou petits enfants en sa compagnie (un peu plus de $70 \%$ ) ou encore d'avoir des rapports sexuels protégés ( $13 \%$ en 2004 contre $17,4 \%$ en 2001), indiquant que cette tolérance ne résiste pas à une forte proximité.

En revanche, de façon constante à travers les enquêtes, les femmes se sont toujours senties davantage concernées par les campagnes de prévention que les hommes : un tiers d'entre elles ont répondu se sentir très concernées par les campagnes d'information (versus $28 \%$ des hommes). Cet intérêt est toutefois plus faible qu'il y a dix ans : $39 \%$ se disaient alors très concernées.

Ce moindre intérêt s'accompagne d'une moindre visibilité de la maladie : les femmes sont moins nombreuses en 2004 qu'en 2001 à déclarer connaître une personne séropositive et moins encore qu'en 1998. Elles étaient près d'une sur trois en 1998, elles sont moins d'une sur quatre en 2004. De plus, alors qu'en 1998 il s'agissait dans $85,2 \%$ des cas d'une connaissance proche, c'està-dire un parent, un ami, un collègue de travail ou un partenaire sexuel, ce pourcentage n'est que de 74,7\% en 2004 .

La baisse de proximité et de visibilité de la maladie s'observe particulièrement chez les femmes les moins diplômées. Celles qui ne possèdent pas le baccalauréat expriment également plus souvent une plus faible acceptation des personnes séropositives et sont moins nombreuses à avoir déjà craint d'être contaminées : $16 \%$ des femmes sans diplôme versus $37 \%$ des femmes possédant un diplôme supérieur au baccalauréat ont déjà craint d'avoir été contaminées par le virus du sida.

\section{Risques individuel et social de la contamination : vers une normalisation du sida chez les femmes comme chez les hommes}

L'essoufflement de la mobilisation résulte-t-il d'une moindre perception subjective du risque de contamination?

\section{Risque individuel}

La proximité à la maladie et la perception du risque individuel de contamination par le virus du sida sont liées chez les femmes, comme chez les hommes. Celles qui connaissent un parent, un ami, un collègue ou un partenaire séropositif sont plus nombreuses à avoir déjà craint d'avoir été contaminées par le virus du sida : $36,9 \%$ contre $28,2 \%$ parmi celles qui ne connaissent pas dans leur entourage proche une personne séropositive.

Ce lien entre proximité et perception du risque individuel semble toutefois moins fort dans la dernière enquête. En effet, les femmes déclarent moins fréquemment connaître une personne séropositive en 2004 qu'en 1998, mais elles n'ont pas pour autant une perception plus faible du risque de contamination. La proportion de femmes ayant une perception supérieure ou égale à la moyenne d'être contaminées par le virus du sida est stable et celles-ci sont même plus nombreuses en 2004 à avoir déjà craint une contamination : 29,6\% versus environ $25 \%$ en 2001 et en $1998(p<0,02)$.

Les mêmes résultats sont observés chez les hommes. La crainte d'avoir soi-même déjà été contaminé est en revanche stable pour les hommes entre 1998 et 2004 et ils restent ainsi proportionnellement moins nombreux que les femmes à rapporter cette peur.

\section{Risque social}

La perception du risque social est appréhendée dans l'enquête à travers les données sur la crainte de différents risques et maladies $^{3}$. La comparaison du niveau de crainte de ces maladies et leur évolution au fil des enquêtes indiquent une réduction du risque social perçu : la crainte du sida diminue depuis 1994, passant de $35,6 \%$ à $14,6 \%$ en 2004 pour les femmes, et respectivement de $33,3 \%$ à $13,4 \%$ pour les hommes. Du fait de cette forte diminution, les maladies liées à l'alcool et au tabac, les maladies cardiaques et les accidents de la circulation sont aujourd'hui beaucoup plus redoutés que le sida.

Le sida apparait donc aujourd'hui comme une maladie chronique. Cette normalisation sociale constatée chez les femmes concerne aussi les hommes. Certes, les femmes rapportent un plus grand intérêt pour les campagnes d'information sur le sida et une crainte individuelle de la contamination un peu plus élevée que les hommes, mais le niveau de connaissance des modes de transmission et des moyens de protection, la proximité aux malades et la perception du risque social, ainsi que leurs évolutions depuis 1992 sont assez similaires.

-Parmi ces différents risques et maladies, pouvez-vous me dire si vous les craignez pour vous-méme, pas du tout, peu, pas mal, beaucoup ”. 


\section{Des comportements sexuels et préventifs différents chez les femmes et chez les hommes}

La normalisation sociale du sida constatée depuis 1998 semble s'accompagner depuis 2001 d'un relâchement des comportements de prévention, laissant entrevoir des liens entre représentation sociale, perception du risque et adoption de comportements préventifs. Ces liens sont complexes. Une bonne connaissance de la maladie et une forte perception des risques individuel et social ne sont pas des conditions suffisantes pour conduire à l'adoption de comportements préventifs. En effet, alors que les femmes et les hommes participent de la même façon au processus de normalisation du sida, les comportements sexuels et préventifs diffèrent entre eux.

Plusieurs questions permettent, dans l'enquête KABP, de décrire les comportements sexuels et préventifs. Deux indicateurs, rendant compte des situations à risque, sont plus particulièrement analysés (tableau 3) : le multipartenariat, défini par le fait d'avoir deux partenaires ou plus au cours des douze derniers mois et la non-utilisation du préservatif au cours des douze derniers mois, au moment de l'entrée dans la vie sexuelle et des premiers rapports sexuels avec un nouveau partenaire.

Quelle que soit l'année de l'enquête, les femmes sont toujours davantage abstinentes et déclarent moins de partenaires que les hommes. En 2004, 8 \% des femmes déclaraient avoir eu plusieurs partenaires au cours de l'année, proportion stable depuis 1994. Cette proportion est de $14 \%$ chez les hommes, en augmentation depuis 2001 (10\%). Les femmes, comme les hommes, déclaraient plus fréquemment de nouveaux partenaires en 2004 qu'en 2001 : parmi les femmes qui ont eu au moins un partenaire dans l'année, $15 \%$ en ont déclaré au moins un nouveau, versus $11 \%$ en 2001. Ce renouvellement de partenaires (plusieurs partenaires et plus souvent des nouveaux) est plus fréquent parmi les jeunes femmes et les jeunes hommes âgés de 18-29 ans, tranche d'âge correspondant à une période de recherche de partenaires. Alors que ce comportement ne dépend pas du tout du diplôme chez les hommes (quelle que soit l'année de l'enquête), il est plus fréquent parmi les femmes plus diplômées : ainsi en 2004, 9,7\% des femmes possédant au moins le baccalauréat déclaraient avoir eu plusieurs partenaires au cours des douze derniers mois et $15,9 \%$ au moins un nouveau ; elles sont respectivement $5,6 \%$ et $10,1 \%$ parmi les moins diplômées.

Les femmes utilisent aujourd'hui largement le préservatif à l'entrée dans la sexualité ou au début d'une nouvelle relation. Ainsi, plus de trois quarts d'entre elles qui ont commencé leur vie sexuelle entre 1998 et 2000 déclarent l'avoir utilisé au moment de leur premier rapport sexuel ou avec un nouveau partenaire. Mais cette utilisation reste plus faible que chez les hommes, puisqu'ils sont un peu plus de $80 \%$ à déclarer l'avoir utilisé dans ces situations.

Elles sont aussi plus nombreuses que les hommes à ne pas l'avoir utilisé au cours de l'année précédente, dans des situations où elles déclarent plusieurs partenaires (tableau 3). Cette proportion

Tableau 3

Nombre de partenaires sexuels et non-utilisation du préservatif au cours de l'année chez les femmes et chez les hommes - enquêtes KABP 1994, 1998, 2001 et 2004

\begin{tabular}{|c|c|c|c|c|c|c|c|c|c|c|}
\hline & \multicolumn{5}{|c|}{ Femmes âgées de 18 à 54 ans } & \multicolumn{5}{|c|}{ Hommes âgés de 18 à 54 ans } \\
\hline & 1994 & 1998 & 2001 & 2004 & Proba' ${ }^{1}$ & 1994 & 1998 & 2001 & 2004 & Proba $^{1}$ \\
\hline \multicolumn{11}{|c|}{ Activité sexuelle au cours des 12 derniers mois (\%) } \\
\hline Abstinents & 7,6 & 12,1 & 9,9 & 10 & & 6,8 & 5,8 & 8,7 & 7,2 & \\
\hline Un seul partenaire & 85,7 & 80,6 & 83,8 & 82 & & 75,9 & 79,9 & 80,8 & 78,7 & \\
\hline Plusieurs & 6,7 & 7,3 & 6,3 & 8 & NS & 17,3 & 14,3 & 10,5 & 14,1 & $\mathrm{~s}$ \\
\hline \multicolumn{11}{|c|}{ Nombre de nouveaux partenaires dans les 12 mois (parmi la population ayant déclaré avoir eu un rapport sexuel dans les 12 mois) (\%) } \\
\hline Pas de nouveaux & $86,1^{2}$ & 87 & 88,6 & 84,9 & & 76,9 & 80,1 & 83,3 & 79,1 & \\
\hline Un nouveau & 10,8 & 9,6 & 9,8 & 10,4 & & 12,5 & 12,6 & 9,9 & 13 & \\
\hline Plusieurs nouveaux & 3,1 & 3,4 & 1,6 & 4,7 & $\mathrm{~s}$ & 10,6 & 7,2 & 6,8 & 7,9 & NS \\
\hline \multicolumn{11}{|c|}{ Non-utilisation du préservatif au cours des 12 derniers mois (\%) } \\
\hline Ensemble & 72 & 69,5 & 74,1 & 69,3 & NS & 63,9 & 62,9 & 70,6 & 64,8 & $\mathrm{~S}$ \\
\hline Selon le nombre de $p$ & & & & & & & & & & \\
\hline \multicolumn{11}{|c|}{ - Un partenaire ancien (relation commencée il y a plus d'un an) } \\
\hline \multirow{2}{*}{\multicolumn{11}{|c|}{ - Un partenaire nouveau (relation commencée il y a moins d'un an) }} \\
\hline & & & & & & & & & & \\
\hline \multirow{2}{*}{ - Plusieurs partenaires } & 49,8 & 21,3 & 33,8 & 21,9 & $\mathrm{~s}$ & 38,6 & 24,5 & 34,3 & 17,9 & NS \\
\hline & 19.9 & 19,9 & 31,3 & 29,9 & s & 19,8 & 10,6 & 24,1 & 20,2 & NS \\
\hline
\end{tabular}

1 Test du $\chi 2$ simple sur les effectifs redressés, NS non significatif, $S$ significatif pour un $p<0,05$.

${ }^{2}$ Lecture : En 1994, 86,1\% des femmes âgées de 18 à 54 ans ont déclaré ne pas avoir eu de nouveaux partenaires au cours des 12 demiers mois.

${ }^{3}$ En $1994,78,4 \%$ des femmes âgées de 18 à 54 ans, qui ont commencé leur relation avec leur partenaire il y a plus d'un an, n'ont pas utilisé de prẻservatif dans les 12 derniers mois. 
Tableau 4

Déterminants de la non-utilisation du préservatif au cours de l'année précédant l'enquête parmi les femmes et les hommes déclarant plus d'un partenaire dans l'année

Enquêtes KABP 1994, 1998, 2001 et 2004

\begin{tabular}{|c|c|c|c|c|}
\hline & \multicolumn{2}{|c|}{ Femmes } & \multicolumn{2}{|c|}{ Hommes } \\
\hline & OR & IC à $95 \%$ & OR & IC à $95 \%$ \\
\hline \multicolumn{5}{|l|}{ Année d'enquête } \\
\hline 1992 & 1 & & 1 & \\
\hline 1994 & 0,1 & $0,02-0,5$ & 1,4 & $0,6-3,2$ \\
\hline 1998 & 0,3 & $0,1-0,6$ & 0,3 & $0,1-0,7$ \\
\hline 2001 & 0,5 & $0,2-0,96$ & 0,7 & $0,4-1,4$ \\
\hline 2004 & 0,4 & $0,2-0,8$ & 0,7 & $0,3-1,3$ \\
\hline \multicolumn{5}{|l|}{ Âge à l'enquête } \\
\hline $18-19$ ans & 1 & & 1 & \\
\hline $20-29$ ans & 2,5 & $0,7-9,3$ & 9,4 & $1-84,2$ \\
\hline $30-39$ ans & 2,7 & $0,7-11,1$ & 15,6 & $1,8-135$ \\
\hline $40-49$ ans & 10,2 & $2,6-40,2$ & 29,6 & $3,3-264,4$ \\
\hline $50-54$ ans & 28,4 & $4,2-193,9$ & 47,3 & $4,6-483,5$ \\
\hline \multicolumn{5}{|l|}{ Niveau de diplôme } \\
\hline Aucun & 1 & & 1 & \\
\hline CEP/CAP/BEP & 0,3 & $0,1-0,9$ & 0,95 & $0,4-2,1$ \\
\hline BAC & 0,2 & $0,1-0,7$ & 0,6 & $0,2-1,4$ \\
\hline Supérieur au BAC & 0,1 & $0,04-0,5$ & 0,6 & $0,3-1,4$ \\
\hline \multicolumn{5}{|c|}{ Score d'acceptation des personnes séropositives } \\
\hline Faible acceptation & 1 & & 1 & \\
\hline Forte acceptation & 1,7 & $0,7-4$ & 0,7 & $0,4-1,1$ \\
\hline \multicolumn{5}{|c|}{ Connaissance d'un parent, d'un ami, d'un collègue ou d'un partenaire séropositif } \\
\hline Oui & 1 & & 1 & \\
\hline Non & 1,1 & $0,6-2$ & 1,95 & $1-3,7$ \\
\hline \multicolumn{5}{|c|}{ Score de connaissance des modes incertains de transmission du virus du sida } \\
\hline Faible croyance en ces modes de transmission & 1 & & 1 & \\
\hline Forte croyance en ces modes de transmission & 1,9 & $1,1-3,6$ & 1,3 & $0,8-2$ \\
\hline \multicolumn{5}{|c|}{ Score de connaissance des modes certains de transmission du virus du sida } \\
\hline Modes de transmission peu connus & 1 & & 1 & \\
\hline Modes de transmission connus & 0,9 & $0,5-1,5$ & 1 & $0,6-1,6$ \\
\hline \multicolumn{5}{|c|}{ Perception du risque par rapport à la moyenne des gens } \\
\hline Inférieur ou nul & 1 & & 1 & \\
\hline Egal ou supérieur & 0,9 & $0,5-1,5$ & 0,9 & $0,5-1,5$ \\
\hline
\end{tabular}

Champs : femmes et hommes âgés de 18 à 54 ans déclarant plus d'un partenaire au cours des douze derniers mois

de non-utilisatrices a même augmenté au cours des dix dernières années, puisqu'en 2004 elle était proche de $30 \%$, alors qu'elle était de $20 \%$ en 1994 et en 1998. On observe une tendance similaire, quoique moins accentuée, chez les hommes. Alors que les femmes multipartenaires âgées de 40 à 54 ans restent à un niveau élevé de non-protection (un peu plus de $47 \%$ en 2004), la diminution de l'utilisation du préservatif provient essentiellement des jeunes femmes multipartenaires : elles étaient moins de $9 \%$ en 2001 et en 1998 à déclarer n'avoir utilisé de préservatif avec aucun de leurs partenaires au cours des douze derniers mois, alors qu'elles sont $26 \%$ en 2004. Par ailleurs, ce sont les multipartenaires non diplômées qui sont les plus nombreuses à ne se protéger avec aucun de leurs partenaires : 50,2\% d'entre elles déclarent ne pas avoir utilisé de préservatifs versus $22,4 \%$ des femmes diplômées du supérieur ayant eu plusieurs partenaires au cours des douze derniers mois. En revanche, la non-protection n'est pas liée au diplôme chez les hommes.

Comme le montre le tableau 4, la non-utilisation du préservatif augmente avec l'âge chez les femmes comme chez les hommes, et est plus fréquente chez les femmes non diplômées, ainsi que chez celles qui pensent plus fréquemment que la transmission est possible lors de séances d'acupuncture, lors de soins dentaires et en utilisant le rasoir mécanique d'une personne séropositive. Chez les hommes ayant eu plusieurs partenaires au cours de l'année, connaître un parent, un ami, un collègue ou un partenaire séropositif diminue la probabilité de n'avoir utilisé un préservatif avec aucun de ses partenaires. Chez les femmes, seule une perception plus élevée que la moyenne du risque de 
contamination apparaît comme un facteur associé à l'utilisation du préservatif au cours des douze derniers mois.

Depuis leur début, les enquêtes KABP ont confirmé les relations entre les croyances sur les modes de transmission du VIH et les attitudes face aux personnes atteintes.

L'acceptation des personnes séropositives augmente avec une meilleure connaissance des modes de transmission. En revanche, elle diminue si la croyance est que la transmission du $\mathrm{VIH}$ s'effectue au cours de circonstances incertaines ${ }^{4}$, et ce chez les femmes comme chez les hommes. Pour autant, ces relations ne sont retrouvées que très partiellement au niveau des comportements. Les femmes, comme les hommes, persistent à s'exposer au risque, en dépit d'une bonne connaissance des modes de transmission, en particulier les moins diplômées.

\section{L'adaptation au risque de contamination diffère entre les femmes et les hommes}

Les résultats ont montré que l'exposition au risque, ici essentiellement à travers la non-utilisation du préservatif par les personnes ayant plus d'un partenaire au cours de l'année, est différente chez les femmes et chez les hommes, alors qu'ils partagent une même représentation sociale de la maladie. La perception du risque individuel des femmes et, notamment, de celles déclarant plus d'un partenaire au cours des douze derniers mois, est stable ces dernières années, alors que l'on constate chez elles dans le même temps un relâchement des comportements de prévention. La perception du risque du $\mathrm{VIH}$ n'est donc pas le seul déterminant à l'adoption des comportements de prévention des femmes.

\section{L'adoption de stratégies alternatives à l'usage du préservatif plus fréquente chez les femmes}

Face au risque de transmission sexuelle du VIH, l'utilisation du préservatif ne représente pas toujours la seule et unique réponse en termes de prévention. Sans reprendre ici les éléments avancés, comme par exemple ceux de la théorie économique [7], pour expliquer les raisons qui conduisent les femmes et les hommes à ne pas choisir d'utiliser uniquement le préservatif, nous allons simplement montrer que les femmes adoptent plus fréquemment que les hommes des stratégies basées sur le choix ou la réduction du nombre de partenaires.

En 2001, une majorité de femmes et d'hommes, dans des proportions très proches, déclarent que les stratégies ${ }^{5}$ centrées sur l'idée

\footnotetext{
C'est-à-dire les modes de transmission pour lesquels les risques de contamination ne sont pas avérés (au cours de soins dentaires, en utilisant le rasoir mécanique d'une personne contaminée, lors de séances d'acupuncture).

5 Dans le questionnaire de 2001, il était demandé aux femmes et aux hommes si les situations suivantes correspondaient à leur sexualité actuelle : a je suis fidèle », « je limite le nombre de mes partenaires ", « $j$ 'ai des rapports sexuels uniquement avec des personnes que je connais ", "j'ai arrêté d'avoir des rapports sexuels ", "j'ai abandonné les rapports sexuels avec pénétration ”, "je pose des questions à mon partenaire sur sa vie sexuelle passée ", " je demande un test de dépistage du sida à tout nouveau partenaire ".
}

d'un choix ou d'une réduction du nombre de partenaires, correspondent à leur sexualité actuelle : $96,8 \%$ des femmes et $93,8 \%$ des hommes déclarent être fidèles, $96 \%$ et $92,3 \%$ déclarent limiter le nombre de leurs partenaires et $89,3 \%$ et $87,3 \%$ n'avoir des rapports sexuels qu'avec des personnes qu'ils connaissent.

Limitation du nombre de partenaires et fidélité sont des comportements partagés par l'ensemble des femmes, quel que soit leur âge, les autres situations concernant davantage les plus jeunes d'entre elles. Ainsi, les femmes âgées de moins de 40 ans sont environ $92 \%$ à répondre n'avoir des rapports sexuels qu'avec des personnes connues (versus moins de $86 \%$ chez les plus âgées) et elles sont près d'une sur quatre à demander un test de dépistage à tout nouveau partenaire (contre moins d'une sur sept).

Alors que l'adoption de ces stratégies n'apparaît pas liée au niveau de diplôme chez les hommes, les femmes les plus diplômées choisissent davantage que les moins diplômées les stratégies basées sur la réduction du nombre de partenaires (limiter le nombre de ses partenaires, n'avoir des rapports sexuels qu'avec des personnes connues), ces dernières rapportant plus souvent l'abandon de la pénétration, voire l'arrêt des rapports sexuels.

Les stratégies centrées sur la réduction du nombre de partenaires ne se substituent pas totalement à l'utilisation du préservatif, puisqu'elles sont aussi bien citées par les femmes qui déclarent avoir utilisé des préservatifs au cours des douze derniers mois que par celles qui disent ne pas en avoir utilisé : $93,4 \%$ des femmes ayant utilisé un préservatif au cours des douze derniers mois déclarent être fidèles à leur partenaire et $94,8 \%$ déclarent limiter le nombre de leurs partenaires. Elles se présentent comme des stratégies complémentaires.

Chez les hommes, ces stratégies de réduction du nombre de partenaires sont plus fréquentes parmi ceux qui déclarent ne pas avoir utilisé de préservatif dans l'année, ce qui est en faveur de leur substitution au préservatif.

Enfin, l'adoption de ces stratégies alternatives n'est pas liée à la perception du risque individuel de contamination, puisque les femmes ayant une perception égale ou supérieure à la moyenne sont aussi nombreuses que celles chez qui elle est plus faible à déclarer être fidèles, à limiter le nombre des partenaires, à avoir des rapports sexuels uniquement avec des personnes connues ou encore à demander un test de dépistage à tout nouveau partenaire.

Comme pour l'utilisation du préservatif au cours de l'année, se déclarer fidèle, sélectionner ou mieux connaître ses partenaires sexuels, sont, chez les femmes, indépendants de la perception du risque de contamination. L'adoption des comportements de prévention dépasse donc le simple cadre d'une adaptation au risque de contamination et renvoie davantage aujourd'hui à une norme sociale de la sexualité différente chez les femmes et chez les hommes (cf. I'article de Nathalie Bajos et coll, page 24). 


\section{L'importance du cadre relationnel}

Si peu de questions permettent dans les enquêtes KABP de prendre en compte le cadre relationnel (la nature de la relation, les caractéristiques du partenaire, ...), leur analyse souligne que le contexte de la relation et les caractéristiques du partenaire sont des éléments importants dans la négociation du préservatif, particulièrement chez les femmes.

\section{Le cadre relationnel du premier rapport sexuel et du rapport avec le dernier partenaire}

Les sujets ont été interrogés sur leur désir d'avoir leur premier rapport sexuel ${ }^{6}$. Les femmes sont moins nombreuses que les hommes à avoir désiré ce premier rapport $(77,5 \%$ versus $90,8 \%$ des hommes). De plus, celles qui ne le souhaitaient pas ont moins souvent utilisé le préservatif à ce moment-là : $35,4 \%$ versus $44,5 \%$ de celles qui ont désiré ce premier rapport sexuel. Cet écart est essentiellement lié aux femmes ayant une scolarité inférieure au baccalauréat : parmi elles, $36,5 \%$ de celles qui désiraient ce rapport ont déclaré avoir utilisé un préservatif contre $24,6 \%$ parmi celles qui ne le désiraient pas. Ce pourcentage est d'environ 47 \% parmi les femmes titulaires d'un diplôme supérieur, qu'elles aient ou non souhaité ce rapport à ce moment-là.

En revanche, chez les hommes, l'utilisation du préservatif au moment de l'entrée dans la vie sexuelle n'est pas liée au désir du premier rapport : $44 \%$ de ceux qui l'ont désiré ont utilisé un préservatif, contre $41 \%$ de ceux qui ne le souhaitaient pas $(p>0,05)$.

Les femmes, et notamment les plus jeunes et les plus diplômées, sont plus nombreuses que les hommes à avoir abordé différents sujets avant leur premier rapport avec ce dernier partenaire : $60,6 \%$ ont parlé de leur vie sexuelle passée $(49,5 \%$ des hommes), $62,2 \%$ de contraception (48,7\% des hommes), $36,4 \%$ du sida ou des infections sexuellement transmissibles $(30,1 \%$ des hommes) et $52 \%$ de l'utilisation du préservatif ( $47,7 \%$ des hommes). Parmi les femmes titulaires du baccalauréat ou d'un diplôme du supérieur, $57,5 \%$ disent avoir abordé la question de l'utilisation du préservatif (contre $43,6 \%$ des moins diplômées) et $64,1 \%$ ont parlé de leur vie sexuelle passée (contre $55,4 \%$ des moins diplômées).

Les jeunes femmes, qui ont une perception du risque de contamination par le VIH plus élevée que la moyenne des gens, parlent davantage avec leur partenaire de leur vie sexuelle, des maladies sexuellement transmissibles et du préservatif que celles qui ont une perception moins élevée, la contraception étant abordée quelle que soit leur perception du risque de contamination.

Les femmes et les hommes qui ont commencé une relation avec un partenaire il y a moins de douze mois, c'est-à-dire qui se sont

\footnotetext{
* Ce premier rapport sexuel était-t-il quelque chose que vous souhaitiez à ce moment là, que vous avez accepté, mais que vous ne souhaitiez pas vraiment, que vous avez èté forcé(e) de faire contre votre volonté? *
}

engagés dans une nouvelle relation, sont respectivement $54,8 \%$ et $72,5 \%$ à déclarer avoir utilisé un préservatif lors du dernier rapport avec ce partenaire. Chez les femmes, il est plus fréquemment utilisé lorsque ce nouveau partenaire est considéré comme un partenaire occasionnel que comme un partenaire régulier : $74,6 \%$ contre $51,2 \%(p=0,03)$, ce qui n'est pas le cas chez les hommes.

Le cadre relationnel du rapport sexuel apparaitt particulièrement important chez les femmes. Elles se retrouvent plus souvent que les hommes à accepter un premier rapport qu'elles ne souhaitent pas vraiment, situation qui rend plus difficile l'utilisation du préservatif, alors même qu'elles disent aborder plus fréquemment le sujet de la contraception, des infections sexuellement transmissibles et celui de l'utilisation du préservatif lorsqu'elles commencent une nouvelle relation. Notons toutefois que les femmes qui ont une perception élevée du risque de contamination abordent plus souvent ces sujets et déclarent plus souvent avoir utilisé un préservatif.

\section{La négociation du préservatif en cas de refus d'utilisation}

Pour la première en fois en 2004, il était demandé aux personnes répondant à l'enquête si elles avaient déjà refusé d'utiliser un préservatif avec un partenaire qui le souhaitait ou si leur partenaire avait déjà refusé un préservatif qu'elles/eux-mêmes proposaient.

Sur l'ensemble des personnes ayant déjà eu un rapport sexuel, moins de $3 \%$ des femmes et des hommes déclarent avoir refusé d'utiliser un préservatif avec un de leur partenaire dans les cinq dernières années. Une minorité plus importante déclare avoir été confrontée dans les cinq dernières années à un refus de l'un de leurs partenaires. Cette situation est davantage rencontrée par les femmes que par les hommes ( $8,7 \%$ versus $5,3 \%, p<0,05)$ et par les plus jeunes: $19 \%$ des femmes entre 18 et 24 ans (13,4\% des hommes du même âge), $12,2 \%$ des femmes de $25-34$ ans (6,4 \% des hommes) ; elles sont moins de $3 \%$ parmi les plus âgées.

En gardant une grande prudence liée à la faiblesse des effectifs, il semble que la gestion de ce refus tend de plus à être différente pour les femmes et les hommes, les femmes se retrouvant plus souvent à devoir accepter un rapport sexuel sans préservatif.

Lorsque leur partenaire a refusé d'utiliser un préservatif, $55,3 \%$ des femmes (43,5\% des hommes) disent avoir eu le rapport sans préservatif, $17,4 \%$ sans pénétration et $16,2 \%$ avec un préservatif. Moins d'un tiers d'entre elles (29,7 \%) et $40,8 \%$ des hommes $(p=0,2)$ disent avoir renoncé à ce rapport.

Les plus jeunes, hommes ou femmes, déclarent plus fréquemment renoncer au rapport sexuel ; les plus âgés, surtout les femmes, céderaient davantage en acceptant le rapport sans préservatif. Notons que si $54 \%$ des jeunes hommes y renoncent et $17 \%$ 
Tableau 5

Comportements des femmes et des hommes face au refus d'un partenaire d'utiliser le préservatif selon l'âge, le diplôme et le nombre de partenaires déclarés dans les cinq dernières années

\begin{tabular}{|c|c|c|c|c|c|c|c|c|}
\hline & \multicolumn{4}{|c|}{ Femmes } & \multicolumn{4}{|c|}{ Hommes } \\
\hline & $\begin{array}{l}\text { A renoncé } \\
\text { au rapport }\end{array}$ & $\begin{array}{l}\text { Rapport sans } \\
\text { pénétration }\end{array}$ & $\begin{array}{c}\text { Rapport sans } \\
\text { préservatif }\end{array}$ & $\begin{array}{c}\text { A utilisé } \\
\text { le préservatif }\end{array}$ & $\begin{array}{l}\text { A renoncé } \\
\text { au rapport }\end{array}$ & $\begin{array}{l}\text { Rapport sans } \\
\text { pénétration }\end{array}$ & $\begin{array}{c}\text { Rapport sans } \\
\text { préservatif }\end{array}$ & $\begin{array}{l}\text { A utilisé le } \\
\text { préservatif }\end{array}$ \\
\hline Effectif & 163 & 147 & 130 & 120 & 85 & 71 & 68 & 62 \\
\hline Ensemble & 29,7 & 17,4 & 55,3 & 16,2 & 40,8 & 10,5 & 43,5 & 26,6 \\
\hline $\begin{array}{l}\text { Âge } \\
18-24 \text { ans } \\
25-39 \text { ans } \\
40-54 \text { ans } \\
\text { Probabilité } 1\end{array}$ & $\begin{array}{r}40,1 \\
25,9 \\
19,4 \\
\text { NS }\end{array}$ & $\begin{array}{r}12,4 \\
21 \\
17,3 \\
\text { NS }\end{array}$ & $\begin{array}{r}44,6 \\
56,9 \\
72,2 \\
\text { NS }\end{array}$ & $\begin{array}{r}19,5 \\
15,7 \\
10,3 \\
\text { NS }\end{array}$ & $\begin{array}{r}53,8 \\
33,1 \\
20,2 \\
\text { NS }\end{array}$ & $\begin{array}{r}16,3 \\
7,5 \\
0 \\
\text { NS }\end{array}$ & $\begin{array}{r}17,1 \\
61,7 \\
59,5 \\
\mathrm{~S}\end{array}$ & $\begin{array}{r}36,7 \\
18,1 \\
19,8 \\
\text { NS }\end{array}$ \\
\hline $\begin{array}{l}\text { Diplôme } \\
<\text { BAC } \\
\text { BAC et + } \\
\text { Probabilité1 }\end{array}$ & $\begin{array}{r}32,6 \\
28,4 \\
\text { NS }\end{array}$ & $\begin{array}{r}21,3 \\
15,9 \\
\text { NS }\end{array}$ & $\begin{array}{r}55,2 \\
55,3 \\
\text { NS }\end{array}$ & $\begin{array}{r}20,8 \\
14,4 \\
\text { NS }\end{array}$ & $\begin{array}{r}48,7 \\
33,3 \\
\text { NS }\end{array}$ & $\begin{array}{r}11,9 \\
9,3 \\
\text { NS }\end{array}$ & $\begin{array}{r}33,9 \\
51,5 \\
\text { NS }\end{array}$ & $\begin{array}{l}31 \\
23 \\
\text { NS }\end{array}$ \\
\hline $\begin{array}{l}\text { Selon le nor } \\
\text { Un seul } \\
\text { Plusieurs } \\
\text { Probabilité }\end{array}$ & $\begin{array}{c}\text { tenaires au c } \\
20,1 \\
34,4 \\
\text { NS }\end{array}$ & $\begin{array}{c}\text { Irs des cinq der } \\
23,6 \\
14,4 \\
\text { NS }\end{array}$ & $\begin{array}{c}\text { ères années } \\
68,5 \\
49,9 \\
\text { NS }\end{array}$ & $\begin{array}{r}18,1 \\
15,4 \\
\text { NS }\end{array}$ & $\begin{array}{r}30,9 \\
43,3 \\
\text { NS }\end{array}$ & $\begin{array}{r}4,8 \\
11,7 \\
\text { NS }\end{array}$ & $\begin{array}{r}67,2 \\
37,6 \\
\text { NS }\end{array}$ & $\begin{array}{r}16,4 \\
28,6 \\
\text { NS }\end{array}$ \\
\hline
\end{tabular}

Champs : femmes et hommes qui ont déjà eu un rapport sexuel et qui ont été confrontés dans les cinq dernières années à un refus par leur partenaire d'utiliser un préservatif.

${ }^{1}$ Test du $\chi 2$ simple sur les effectifs redressés ; NS non significatif ; S significatif pour un $p<0,05$.

disent ne pas avoir utilisé de préservatif lors de ce rapport sexuel, les jeunes femmes sont à peu près aussi nombreuses à renoncer ou à accepter ce rapport sans utiliser de préservatif : un peu plus de $40 \%$ des femmes âgées de 18 à 24 ans (tableau 5).

Parmi les femmes qui ont eu plus d'un partenaire au cours des cinq dernières années, une sur deux, confrontée à un refus de son partenaire, déclare avoir eu le rapport sans préservatif contre $37,6 \%$ des hommes.

Ces résultats suggèrent que la négociation du préservatif est plus complexe pour les femmes que pour les hommes, les femmes étant plus fréquemment que les hommes confrontées à un refus. De plus, les hommes ayant refusé d'utiliser un préservatif restent plus nombreux que les femmes à avoir poursuivi le rapport sans protection, mais moins nombreux à avoir subi ce choix si c'est leur partenaire qui ne voulait pas utiliser de préservatifs.

\section{Discussion}

Les résultats des enquêtes KABP réalisées en France depuis plus de dix ans indiquent, une nouvelle fois, que les liens entre connaissance de la maladie, perception du risque et adoption de comportements de prévention sont complexes.

Les femmes ont, comme les hommes, une bonne maîtrise des modes de contamination et des moyens de protection, une forte acceptation des personnes séropositives, même si cette acceptation continue de résister à une trop forte proximité. Elles ont une perception du risque de contamination plus élevée que les hommes, mais cette perception, stable au cours des dernières années, s'accompagne dans le même temps d'une moindre protection.

Par ailleurs, malgré une représentation sociale du sida très similaire chez les femmes et les hommes, la perception du risque est légèrement supérieure pour les premières et les comportements de prévention apparaissent différents. Les femmes sont moins nombreuses à déclarer avoir eu plusieurs partenaires au cours de l'année et à avoir eu au moins un nouveau partenaire au cours de cette période. Mais elles rapportent plus fréquemment ne pas avoir utilisé le préservatif dans ces situations.

Ces éléments soulignent les difficultés de certaines femmes à imposer aujourd'hui l'utilisation du préservatif. Elles sont plus fréquemment dans la situation de devoir accepter des rapports sexuels sans préservatif, lorsque leur partenaire ne souhaite pas en utiliser.

Parallèlement, la sélection des partenaires, la réduction de leur nombre, le recours au test de dépistage ou encore, dans une moindre mesure, l'abandon des rapports sexuels ou de la pénétration sont des stratégies adoptées par les femmes pour se prémunir du risque du VIH. Ces stratégies alternatives, plus fréquentes chez les femmes que chez les hommes, ne semblent pas se substituer à l'utilisation du préservatif.

L'analyse de la gestion du risque de contamination, donc de l'adoption de stratégies préventives, doit ainsi tenir compte du cadre relationnel dans lequel se déroule le rapport sexuel qui dessine les inégalités sociales entre les femmes et les hommes. 
Toutefois, les enquêtes KABP n'ont pas pour principal objectif d'étudier la construction sociale des égalités ou inégalités entre les hommes et les femmes, même en matière de connaissance, d'attitudes, de croyance et de comportements face au VIH. Elles montrent cependant que les changements intervenus depuis une quinzaine d'années chez les femmes (amélioration de la connaissance des modes de transmission et de prévention, acceptation des personnes séropositives, perception du risque social et individuel de contamination par le $\mathrm{VIH}$ ) se heurtent aujourd'hui à une normalisation du sida et, également, au rôle des femmes et des hommes dans la sphère de la sexualité, empêchant certaines femmes d'adopter des comportements de prévention adéquats. L'importance du cadre relationnel dans la négociation de l'utilisation du préservatif nous invite à approfondir les liens entre les normes sociales de la sexualité et l'adoption par les femmes des moyens de prévention ou de contraception.

Les résultats présentés ici doivent s'enrichir et être confrontés à d'autres approches pour mieux saisir les facteurs qui contribuent à ce que les femmes adoptent des comportements de prévention et soient à même de les négocier avec leur partenaire masculin. Ils invitent également à mettre en œuvre une politique de prévention qui ait une approche à la fois globale et ciblée. Les incitations à l'adoption de comportements de prévention doivent en effet combiner des facteurs sociaux et économiques et s'adresser, en priorité, aux femmes jeunes et de faible niveau d'éducation qui ont, comme les femmes plus âgées commençant une nouvelle relation, plus de difficultés à adopter ou à faire adopter le préservatif par leur partenaire masculin.

\section{Références bibliographiques}

1. InVS. Lutte contre le VIH/sida et les infections sexuellement transmissibles en France. 10 ans de surveillance, 1996-2005. Rapport. Saint Maurice : InVS, mars 2007.

2. Bouyssou-Michel LA, Semaille C. Surveillance de la syphilis en France, 20002005. Synthèse d'un rapport de l'InVS, octobre 2006 http://www.invs.sante.fr/publications/2006/syphilis_france_2000_2005/ syphilis.pdf dernière consultation le 24/07/07.

3. InVS. Actualisation des données issues de la surveillance de la lymphogranulomatose vénérienne rectale en France. Avril 2002-février 2005, http://www.invs.sante.fr/presse/2005/le_point_sur/lgv_310305/index.htmi, dernière consultation le 24/07/07.

4. Beltzer N, Lagarde M, Wu-Zhou X et al. Les connaissances, attitudes, croyances et comportements face au VIH/sida en Île-de-France : Évolutions 1992-19941998-2001-2004. Paris : ORS d'île-de-France, 2005.

5. Setbon M. L'enquête KABP 2004 confirme-t-elle la normalisation du sida? Transcriptases $2006 ; 126$ : février/mars.

6. Moatti J, Beltzer, Dab W. Les modèles d'analyse des comportements à risque face à l'infection à VIH : une conception trop étroite de la rationalité. Population $1993 ; 5: 1505-1534$.

7. Beltzer N, Moatti JP, Fericelli AM. Choix de stratégies de prévention de l'infection à VIH : une analyse micro-économique. In La sexualité au temps du Sida, N Bajos, M Bozon, A Giami, A Spira et le groupe ACSF, Eds. Paris : PUF, 1998, p. 375-412.

8. Beltzer N, Grémy I et le groupe NEM. Histoire sexuelle d'une relation entre deux partenaires : comment est géré le risque du VIH. Enquête KABP 1998. Rapport de l'ORS île-de-France, mars 2000, 104 p. 